\title{
Low latency IP mobility management: protocol and analysis
}

\author{
Min Liu', Xiaobing Guo ${ }^{2,1,4}$, Anfu Zhou', Shengling Wang ${ }^{1}$, Zhongcheng $\mathrm{Li}^{1}$ and Eryk Dutkiewicz ${ }^{3}$
}

\begin{abstract}
Mobile IP is one of the dominating protocols that enable a mobile node to remain reachable while moving around in the Internet. However, it suffers from long handoff latency and route inefficiency. In this article, we present a novel distributed mobility management architecture, ADA (Asymmetric Double-Agents), which introduces double mobility agents to serve one end-to-end communication. One mobility agent is located close to the MN and the other close to the CN. ADA can achieve both low handoff latency and low transmission latency, which is crucial for improvement of user perceived QoS. It also provides an easy-to-use mechanism for MNs to manage and control each traffic session with a different policy and provide specific QoS support. We apply ADA to MIPv6 communications and present a detailed protocol design. Subsequently, we propose an analytical framework for systematic and thorough performance evaluation of mobile IP-based mobility management protocols. Equipped with this model, we analyze the handoff latency, single interaction delay and total time cost under the bidirectional tunneling mode and the route optimization mode for MIPv6, HMIPv6, CNLP, and ADA. Through both quantitative analysis and NS2-based simulations, we show that ADA significantly outperforms the existing mobility management protocols.
\end{abstract}

\section{Introduction}

Next-generation wireless networks (NGWN) are envisaged to have an all-IP-based infrastructure with the support of heterogeneous wireless access technologies. Mobility management with provision of seamless handoff is crucial for an efficient support of global roaming of mobile nodes in NGWN [1]. Mobility management addresses two main problems: location management and handoff management [2]. Location management enables a network to discover the current point of attachment of mobile terminals for successful information delivery. Handoff management maintains the active connections for roaming mobile terminals as they change their points of attachment to the network.

Mobile IP enables an IP node to maintain its connectivity to the Internet when roaming among different access networks, and is expected to be the main engine for IP layer mobility management in the next generation networks. However, it suffers from long handoff latency and inefficient route problems.

\footnotetext{
* Correspondence: liumin@ict.ac.cn

${ }^{1}$ Institute of Computing Technology, Chinese Academy of Sciences, Beijing,

100190, People's Republic of China

Full list of author information is available at the end of the article
}

\section{Prolonged handoff latency}

Mobile IP requires that a home agent (HA) be notified of every location change of the mobile node (MN). This causes unnecessary signaling overhead and handoff latency, especially for MNs with relatively high mobility and long distance to their HAs. In addition, congestion is likely to arise in the home network and the HA will be the bottleneck point of such congestion.

\section{Inefficient route}

When an $\mathrm{MN}$ moves to a foreign domain, all packets sent to the MN have to be tunneled through its HA along paths that are usually longer than the optimal end-to-end path. The triangular route will cause high transmission delay and congestion in the home network. This problem is especially serious when the MN stays in a remote foreign domain for a long period of time.

Many IP-based micro-mobility management protocols [3-8] have been proposed to reduce handoff latency in mobile IP. Their basic idea is that the majority of user's mobility is local and can be limited in a 'domain' by introducing the notion of hierarchy. Although these solutions achieve reduction in signaling load and handoff latency during movements within one domain, they

\section{SpringerOpen $^{\circ}$}


have high signaling load and long handoff latency for inter-domain roaming. In addition, these protocols cannot alleviate the triangular route problem.

The problem of triangular routing can be solved by route optimization [9]. The basic idea behind route optimization is to use a direct route between MNs and their correspondent nodes (CNs) to bypass the HA. Each $\mathrm{CN}$ maintains an address binding cache of the $\mathrm{MN}$ and tunnels the packets directly to the care-of address $(\mathrm{CoA})$ of the MN. In mobile IPv6 (MIPv6) [10], route optimization has been proposed as a fundamental component, rather than a non-standard set of extensions as in mobile IPv4 (MIPv4) [11]. The major drawback of such a solution is that it also needs the CNs to support routing optimization. In addition, a host needs to differentiate and treat a peer fixed host and a peer mobile host differently. Moreover, route optimization may cause serious security problems.

Although there are many extensions to enhance mobile IP-based mobility management protocols, they often fail to simultaneously solve the prolonged handoff latency and inefficient route problems.

In this article, we present a novel distributed mobility management architecture, ADA, which introduces two asymmetric mobility agents to solve the above two problems. One mobility agent is located close to the MN and acts as a local HA to limit the amount of signaling traffic outside the local domain. The other is located close to the $\mathrm{CN}$ and its major objective is to shorten the distance between the $\mathrm{CN}$ and the MN's HA so as to minimize routing overheads. ADA is proposed for low latency mobility management, including low handoff latency and low transmission latency, which are critical for improvement of user perceived QoS. ADA also makes it possible for MNs to manage and control each traffic session with a different policy based on practical application requirements and network environments. It is also convenient for the $\mathrm{CN}$-located network to monitor and control in-bound and out-bound traffic and provide specific QoS support.

It should be noted that ADA is an extension to the mobile IP-based mobility management architecture and can be applied to both MIPv4 and MIPv6. In this article, we apply ADA to MIPv6 communications and design the corresponding protocol operations. Subsequently, we propose an analytical framework for systematic and thorough performance evaluation of mobile IP-based mobility management protocols. This framework can be used to provide guidelines for decision making of mobility management protocols in various network environments. Equipped with the proposed model, we derive and analyze the handoff latency, single interaction delay, and total time cost for specific application traffic for MIPv6, HMIPv6 [12], CNLP [13], and ADA. We also evaluate the performance gain of these protocols by NS2-based simulations.

The remainder of the article is structured as follows. 'Related work' section offers a brief overview of related work. 'Asymmetric double mobility agents for low latency mobility management' section introduces the basic idea of ADA. 'Application of ADA to mobile IPv6 communications' section applies ADA to MIPv6 communications and presents the detailed protocol design. 'Performance analysis' section proposes an analytical framework for performance evaluation of mobile IP-based mobility management protocols. 'Performance evaluation' section verifies the feasibility and effectiveness of ADA by quantitative analysis and NS2-based simulations. The article is concluded in 'Conclusions' section.

\section{Related work}

One of the research challenges for next generation allIP-based wireless systems is the design of intelligent mobility management techniques that take advantage of IP-based technologies to achieve global roaming among various access technologies [14]. Existing improvement work on mobile IP-based mobility management can be classified into two main categories: (1) those aiming to reduce handoff latency, and (2) those aiming to improve route efficiency.

\section{Approaches to reduce handoff latency}

Hierarchical mobile IP [3] and other micro-mobility protocols such as cellular IP [4], IDMP [5], and HAWAII [6] have been proposed to achieve reduction in handoff latency. These mechanisms introduce another layer of hierarchy to the base MIPv4 architecture to localize the signaling messages to one domain. Hierarchical mobile IP [3] introduces a mobility agent called gateway foreign agent (GFA). When an MN changes a foreign agent (FA) within the same regional network, it does not need to register with its HA. Instead, it performs a regional registration to the GFA to update its CoA. This centralized system architecture is sensitive to the GFAs failure and cause a high traffic load on GFAs [15].

The authors in [7] propose a distributed GFA management scheme where each FA can function either as an ordinary FA or a GFA. Whether an agent should act as an FA or as a GFA depends on user mobility. Thus, the traffic load in a regional network is evenly distributed to each FA. The authors also propose a dynamic scheme which is able to adjust the number of FAs under a GFA for each MN according to the user-variant and time-variant user parameters. In this system, there is no fixed regional network boundary for each $\mathrm{MN}$. An MN decides when to perform a home location update according to its changing mobility and packet arrival pattern. 
The authors in [8] propose another dynamic hierarchical mobility management scheme for MIPv4 networks. In this scheme, when an MN changes its subnet and obtains a new CoA from the new FA, the new FA updates the new address to the MN's previous FA so that the new FA forms a new location management hierarchical level for that user. Packets to be delivered to the MN can be tunneled via the multiple levels of FAs. In order to avoid long packet delivery delays, there is an optimal level number for the hierarchy for each user according to his/her call-to-mobility ratio. The threshold can be dynamically adjusted based on the upto-date mobility and traffic load for each terminal. When the threshold is reached, the MN performs a home registration and sets up a new hierarchy for its further movements.

The authors in [16] present a mailbox-based MIPv4 scheme. A sender sends packets to the receiver's mailbox which will in turn forward them to the destination. During each handoff, a choice can be made on whether to report this handoff to the HA or simply to the mailbox. In this way, the workload on the HA as well as the registration delay can be reduced. When the MN migrates to a foreign network, it sends a registration message to the old FA where its mailbox resides. The old FA then decides whether to move the mailbox to the new FA. Separating the mailbox from its owner can help to enable dynamic tradeoff between the packet delivery cost and the registration cost. The mailbox scheme requires FAs to maintain a large amount of information about MNs. It also calls for the information exchange between the old FA, the new FA, and the HA.

MIPv6 [10] shares many features with MIPv4 [11], but it is integrated into IPv6 and offers many other improvements. In MIPv6, there is no need to deploy special routers as FAs as in MIPv4. As a result, mobility management schemes based on extensions to FAs $[7,8,16]$ cannot work in MIPv6 networks.

HMIPv6 [12] introduces a new Mobile IPv6 node, called the mobility anchor point (MAP), to limit the amount of MIPv6 signaling traffic outside the local domain. An MN entering a MAP domain can bind its current location, on-link care-of address (LCoA), with an address on the MAP's subnet, regional care-of address (RCoA). If the $\mathrm{MN}$ changes its current address within a MAP domain, it only needs to register the new address with the MAP. Hence, only the RCoA needs to be registered with the $\mathrm{CNs}$ and the $\mathrm{HA}$. Although HMIPv6 can help to reduce long handoff latency and excessive signaling traffic associated with MIPv6 during intra-domain handoff, it is not effective when MNs move across MAP domains.

FMIPv6 [17] is another enhancement of MIPv6, which aims to improve handoff latency by delivering packets to the new point of attachment at the earliest opportunity. It does so by obtaining link-layer information (L2 trigger) to forecast handoff events and by enabling the MN to get the new access point and the associated subnet prefix information when the $\mathrm{MN}$ is still connected to its current subnet. FMIPv6 requires information exchange and packets forwarding between the previous access router (PAR) and the new access router (NAR) to reduce handoff latency and packets loss. This requires major modifications to the existing infrastructure.

\section{Approaches to improve route efficiency}

Route optimization [9] has been proposed to alleviate the triangular routing problem in MIPv4 [11]. In MIPv6 [10], route optimization has been proposed as a fundamental component. In the route optimization approach, an $\mathrm{MN}$ is allowed to notify a $\mathrm{CN}$ directly of its current address. Thus, packets from the $\mathrm{CN}$ can be routed directly to the CoA of the MN. However, $\mathrm{CN}$ modification is needed to achieve the optimization, therefore this approach is difficult to deploy. In addition, route optimization may cause serious security problems.

Authors in [18] present a new scheme for reducing link and signaling costs in route optimization. Link and signaling cost functions are introduced to capture the tradeoff between the network resources consumed by the routing path and the signaling and processing load incurred by route optimization. A Markovian decision model is presented in [18] to find an optimal sequence for route optimization.

Authors in [19] address the triangular routing problem by proposing a new entity, temporary home agent (TA), to serve the $\mathrm{MN}$ in foreign networks. When an $\mathrm{MN}$ enters a foreign network, a TA in the foreign network is dynamically selected. The TA allocates a temporary home address (THAddr) for the MN. The MN then uses the THAddr as its source address when initiating new connections. The underlying objective is to shorten the distance between an MN and its HA so as to reduce handoff latency and improve routing efficiency. However, the on-going connections established in previous domains with the old TAs are still served by those TAs. In this case, triangular routes still exist. The proposed TA protocol mainly deals with out-bound connections (from MNs to CNs). For in-bound connections, one may resort to mobile IP.

Authors in [20] propose a session-layer-based mobility architecture called DHARMA, whose aim is to shield the transport layer or application layer protocols from the effects of intermittent connectivity. DHARMA uses the PlanetLab overlay network to select a HA close to the $\mathrm{CN}$ from a distributed set of HAs.

CNLP [13] is a mechanism to achieve simultaneous optimized routing and correspondent node-targeted 
location privacy. In CNLP, a home agent, as specified in MIPv6, is called IP reachability home agent (IRHA) and the home address that is registered at the IRHA is referred to as home address for IP reachability (HoA_IR). In addition to IRHA, CNLP introduces a new entity, optimized routing home agent (ORHA), which is located topologically close to the $\mathrm{CN}$ and is used for optimized communication with this CN. A home address that is registered at the ORHA is called home address for optimized routing (HoA_OR). For mobile node-initiated sessions, the MN uses the ORHA as the home agent and HoA_OR on higher layers. Because the ORHA is near the CN, CNLP reduces the transmission delay and improves route efficiency. However, CNLP cannot improve handoff latency, especially when the $\mathrm{MN}$ is far away from the $\mathrm{CN}$.

\section{Performance evaluation of IP-based mobility management schemes}

Although there has been a lot of research focusing on IP-based mobility management protocols, performance evaluation of these protocols is mainly based on simulation and testbed approaches [21,22]. Also, the scenarios used for simulations in different papers are quite different, thus the comparison of IP-based mobility management protocols is hardly possible.

Little work is available in the literature which assesses IP-based mobility management protocols through analytical models. Current analytical models are often based on simple assumptions and have some drawbacks [1]. Authors in [23] present a simple analytical model to study the handoff latency of IPv6-based mobility protocols within the framework of the EU IST project Moby Dick. Its aim is to assess the most appropriate scheme for its functional specification and implementation.

Signaling load to support mobility (i.e., the bandwidth used by control messages) is analyzed according to binding update (BU) emission frequency in [24].

Authors in [25] analyze the overhead associated with FMIPv6 including the signaling cost and the packet delivery cost. They also compare FMIPv6 with MIPv6 in terms of packet loss rates and buffer requirements. However, handoff latency and the impact of user mobility models are not investigated.

Analytical models for performance evaluation of HMIPv6 in IP-based cellular networks are proposed in [26]. These are based on random-walk and fluid-flow models. Based on the proposed models, the authors analyze the impact of cell residence time and user population on the location update cost and the packet delivery cost.

In [1] the effects of network parameters, such as subnet residence time, packet arrival rate and wireless link delay, are investigated for performance evaluation of
MIPv6, HMIPv6, FMIPv6, and F-HMIPv6 [27] with respect to various metrics such as signaling overhead cost, handoff latency, and packet loss. Numerical results in [1] show that there is a trade-off between these performance metrics and network parameters. However, single interaction delay and total time cost for specific application traffic were not investigated.

\section{Asymmetric double mobility agents for low latency mobility management}

In this section, we present a novel distributed mobility management architecture, ADA (asymmetric doubleagents), which can achieve both low handoff latency and low transmission latency in mobility management.

In this architecture, there are two asymmetric mobility agents to serve one end-to-end communication. One mobility agent is located close to the $\mathrm{MN}$ and is referred to as local mobile proxy (LMP). The other is located close to the $\mathrm{CN}$ and is referred to as correspondent mobile proxy (CMP).

The network architecture shown in Figure 1 illustrates an example of the use of ADA.

The aim of ADA is to enhance performance of Mobile IP while minimizing the impact on mobile IP or other mobile IP based protocols. ADA introduces two new network entities (the LMP and CMP), and minor extensions to the $\mathrm{MN}$ operations. The $\mathrm{CN}$ and HA operations will not be affected. It is pertinent to note that the use of ADA does not rely on, or assume the presence of, a permanent home agent. In other words, a mobile node need not have a permanent home address or home agent in order to be ADA-aware or use the features in ADA.

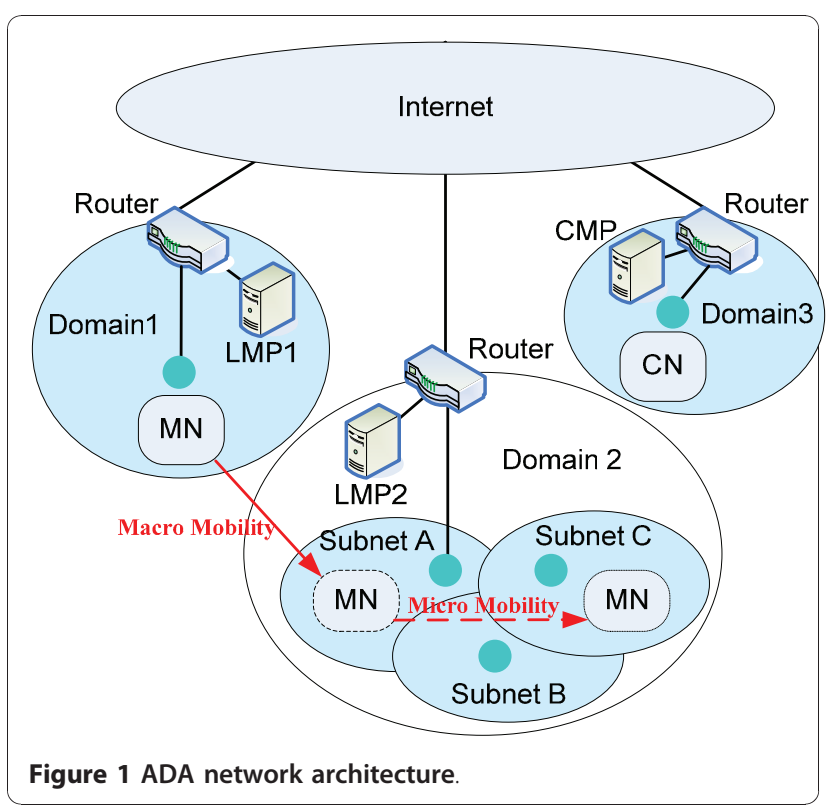


Some important terminologies in ADA are defined as follows.

Local Mobile Proxy (LMP): An LMP is essentially a local home agent. The function of the LMP is like that of the GFA in hierarchical mobile IP [3] and MAP in hierarchical mobile IPv6 [12]. Its aim is to classify the movement of mobile users into macro-mobility and micro-mobility, and provide local mobility handling. By limiting the regional handoff process and signaling traffic to the local domain, the LMP helps to reduce the handoff latency and signaling overhead. Compared with GFA and MAP, the traffic load of the LMP will be alleviated significantly as a result of the CMP's participation. However, the LMP need not have any knowledge of the MN's CMP. In other words, the existence of the CMP is transparent to the LMP. As a result, the LMP can work independently. In this scenario, ADA will degenerate to general hierarchical Mobile IP solution.

Correspondent Mobile Proxy (CMP): A CMP is essentially a locality-optimized home agent. For every communication, ADA dynamically selects a CMP located topologically close to the CN. Like the ORHA in CNLP, the major objective of the CMP in ADA is to shorten the distance between the $\mathrm{CN}$ and the MN's HA so as to minimize routing overheads. However, because of the design aims of ADA (not only to reduce routing overheads but also to shorten handoff latency) and its special double agents architecture, the operation procedure and signaling structure of the CMP are totally different from those in the ORHA. In ADA, the CMP should be able to accept registrations from the $\mathrm{MN}$ that indicate its LMP information.

Distributed home address (DHoA): A DHoA is a home address allocated by the CMP to the MN.

With the function of the LMP, ADA reduces the handoff latency and signaling overhead associated with mobile IP during handoff within one domain. In addition, because the CMP is near the $\mathrm{CN}$, ADA can provide efficient route for all $\mathrm{CNs}$ including those that do not support route optimization.

An ADA-aware MN can choose whether to use the CMP for a connection based on the application type and the expected communication traffic. During the communication with the $\mathrm{CN}$, if the $\mathrm{MN}$ changes its current address, the $\mathrm{MN}$ can decide whether to inform the corresponding CMP to update its CoA based on the requirement of the current communication and the network environment of the new link. The MN can choose to update the bindings in only a certain set of CMPs and thus only maintain the communications with the corresponding CNs. This provides an easy-to-use mechanism for MNs to manage and control each traffic session with a different policy based on practical application requirements and network environments.
Generally, sessions that need to be maintained during the MN's mobility are mainly the client-server types where the CNs are servers. Many applications, such as video on demand, e-mail retrieval, file downloading, fall in this category [19]. ADA is well-suited for this case and can help such CNs enhance their support for their clients' mobility without any change to the CN's implementation. It is also convenient for the $\mathrm{CN}$-located network to monitor and control in-bound and out-bound traffic, and provide specific QoS support. For example, an ISP can set a policy on its CMP to control the maximum number of roaming users. The introduction of the CMP not only allows the shortest communication path to be used, but also eliminates congestion at the MN's HA and home link. In addition, the impact of any possible failures of the HA and home link on the path to or from the MN is reduced.

ADA introduces a small amount of additional state for each CN's CMP, some additional messaging, and a little time delay before it can be turned on for a new connection. However, it is believed that in most cases the benefits far outweigh the costs. In 'Performance evaluation' section, we will evaluate performance of ADA through both quantitative analysis and NS2-based simulations.

\section{Application of ADA to mobile IPv6 communications}

ADA is an extension to the mobile IP-based mobility management architecture and can be applied to both MIPv4 and MIPv6. In this section, we apply ADA to MIPv6 communications and present the detailed protocol design.

In order to keep the maximum compatibility to existing protocols, when applied to MIPv6, the function of the LMP in ADA is the same as that of the MAP in HMIPv6. No signal is added and no operation is changed. As a result, ADA can be compatible with both MIPv6 and HMIPv6. ADA can also work with other micro-mobility protocols. For example, when applied to MIPv4, we can use the GFA as the LMP, so that ADA can be compatible with hierarchical mobile IP either.

Next, we present the detailed operations of ADA when applied to MIPv6 in the bidirectional tunneling (BT) mode and route optimization (RO) mode, respectively. Only the incremental modifications compared with the standard HMIPv6 and MIPv6 protocols are discussed. As the function of the LMP is the same as that of the MAP, in the following description, we will use MAP instead of LMP. The terms RCoA, LCoA, BU, BA, LBU in ADA are defined the same as in HMIPv6. Compared with HMIPv6, there is one type of special binding update in ADA: CMP binding update (CBU). There are two types of CBU: CBU-L and CBU-R. The MN sends a CBU-L to the CMP in order to establish a binding 
between the DHoA and LCoA, while a CBU-R is used to establish a binding between the DHoA and RCoA.

\section{Bidirectional tunneling mode Connection initiation process}

If the $\mathrm{MN}$ wants to initiate a session with ADA support, it should firstly try to discover a CMP in the CN's domain. The process is similar to the mechanism, known as 'location-dependent home agent discovery' in [13]. If the discovery is successful, the MN will bootstrap with the CMP using the mechanisms specified in [28]. During the bootstrapping process, the CMP will establish a binding between the DHoA and LCoA. Then the $\mathrm{MN}$ can communicate with the $\mathrm{CN}$ through the CMP in BT mode. At the same time, the MN will send a CBU-R to the CMP to bind the MN's DHoA to its RCoA. The LCoA is used as the source address of the CBU-R.

Actually, many options can be proposed to implement the discovery of a CMP and the assignment of a DHoA. In this article, we assume the connection initiation process of ADA in BT mode will take two Round-Trip Times (RTTs) between the MN and the CMP.

\section{Intra-domain handoff process}

The intra-domain handoff process of ADA in BT mode is shown in Figure 2, where the broken line indicates that the packet is sent in parallel with the previous one and the gray bold line indicates that the packet is the same as in HMIPv6. In Figure 2 and the following flow diagrams, we indicate the source address of some packets by brackets. For example, (LCoA) data means that the source address of the data is LCoA.

When the MN moves within the same MAP domain, it should send (in parallel) a LBU message to the MAP and a CBU-L message to the CMP to register its new LCoA. In this case, the RCoA remains unchanged. When receiving the $\mathrm{BA}$ from the MAP, the MN can resume its communication with the $\mathrm{CN}$ through the MAP and the CMP. After receiving the BA from the CMP, the MN can communicate with the $\mathrm{CN}$ through the CMP without the transfer of the MAP.

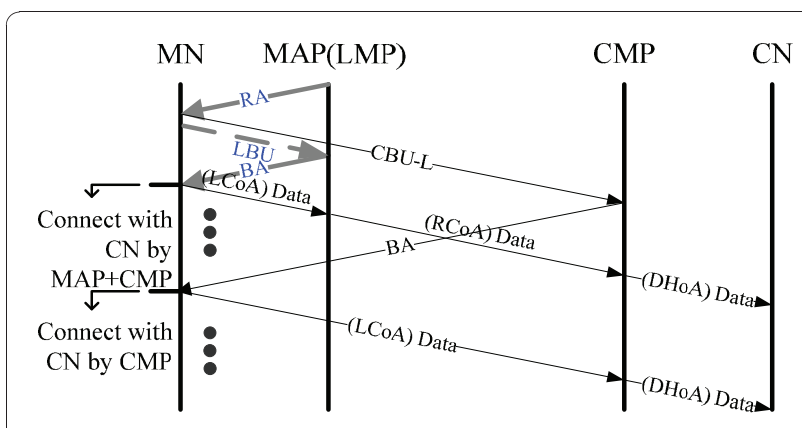

Figure 2 Intra-domain handoff process of ADA in BT mode.

\section{Inter-domain handoff process}

The inter-domain handoff process of ADA in BT mode is shown in Figure 3. The RA used to detect movement will also inform the MN whether it is still in the same MAP domain. If a change in the advertised MAP's address is received, the MN needs to configure two new CoAs: an RCoA on the MAP's link and an LCoA. Then the MN will send the MAP an LBU to bind its RCoA to LCoA. At the same time, a CBU-L will be sent to the CMP to register the MN's new $\mathrm{LCoA}$. When receiving the $\mathrm{BA}$ from the CMP, the MN can resume its communication with the $\mathrm{CN}$ through the CMP. After receiving the BA from the MAP, the MN will register its RCoA with the CMP by sending it a CBU-R.

\section{Route optimization mode}

As the CMP is located close to the $\mathrm{CN}$, ADA can provide an efficient route even in the bidirectional tunneling mode. Thus, although ADA supports the route optimization mode, it is only recommended for communications with a large amount of traffic.

\section{Connection initiation process}

As shown in Figure 4, the connection initiation process of ADA in the RO mode is an extension to the BT mode. In Figure 4, the CMP discovery and bootstrapping processes have been omitted. After bootstrapping, the $\mathrm{MN}$ can communicate with the $\mathrm{CN}$ through the CMP in BT mode. At the same time, the MN will send a CBU-R to the CMP to bind the MN's DHoA to its RCoA. Simultaneously, the MN sends (in parallel) a home test init (HoTI) message through the CMP to the $\mathrm{CN}$ and a care-of test init (CoTI) message directly to the $\mathrm{CN}$. The $\mathrm{CN}$ will respond with home test (HoT) and care-of test (CoT), respectively. Upon successfully completing this return routability (RR) procedure, a $\mathrm{BU}$ will be sent to the CN to register the MN's LCoA. Meanwhile, when the MN receives the BA from the CMP for CBU-R, it will initiate another RR procedure. In this RR procedure, the MN sends a HoTI message through the MAP and the CMP to the $\mathrm{CN}$ and a CoTI

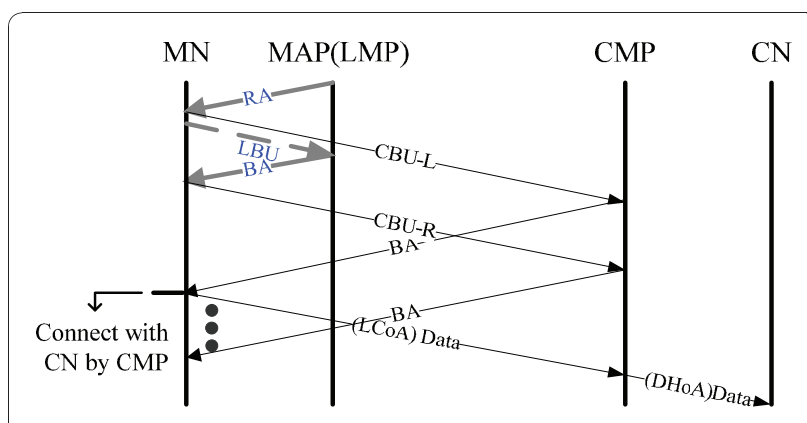

Figure 3 Inter-domain handoff process of ADA in BT mode. 


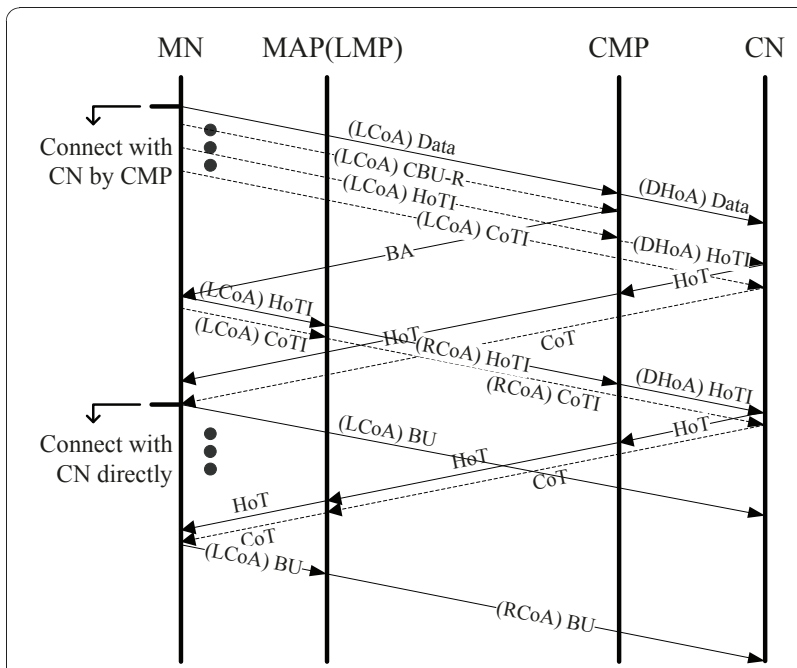

Figure 4 Connection initiation process of ADA in RO mode.

message through the MAP to the $\mathrm{CN}$. After successfully completing this $\mathrm{RR}$ procedure, a $\mathrm{BU}$ will be sent through the MAP to the CN to register the MN's RCoA. Intra-domain handoff process

The intra-domain handoff process of ADA in the RO mode is shown in Figure 5.

In this case, the MN should register its new LCoA with its MAP and CMP. After receiving the BA from the MAP, the MN can resume its communication with the $\mathrm{CN}$ through the MAP. Simultaneously, the MN will send a HoTI message through the MAP to the $\mathrm{CN}$ and a CoTI message directly to the $\mathrm{CN}$. Because the RCoA registered with the $\mathrm{CN}$ remains unchanged, this $\mathrm{RR}$ procedure does not need the participation of the CMP. This RR procedure will be followed by sending a BU to the $\mathrm{CN}$ to register the MN's LCoA.

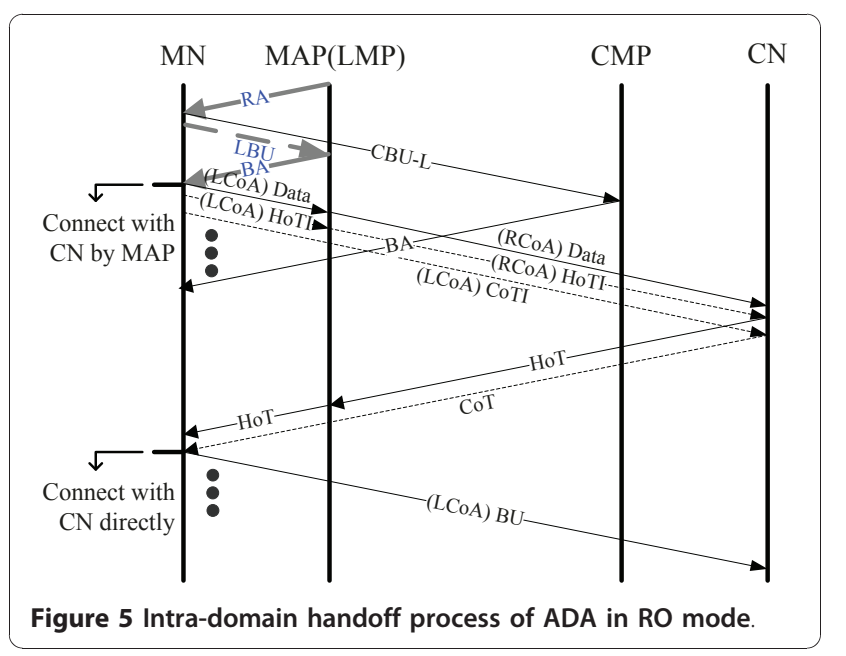

\section{Inter-domain handoff process}

As shown in Figure 6, when an MN moves into a new MAP domain, it should register its new LCoA with its MAP and CMP, respectively. When receiving the BA from the CMP, the MN can resume its communication with the $\mathrm{CN}$ through the CMP. At the same time, the $\mathrm{MN}$ can initiate an RR procedure through the CMP to register its $\mathrm{LCoA}$ with the $\mathrm{CN}$.

After registering with the MAP, the MN should register its new RCoA with its CMP. Upon receiving the BA from the CMP, the MN will initiate an RR procedure through the MAP and the CMP to register its RCoA with the $\mathrm{CN}$.

\section{Performance analysis}

Analytical framework

In this section, we develop an analytical model to evaluate the mobile IP-based protocols. In our model CNs are fixed nodes and multihomed environments are not considered.

As shown in Figure 7, there are $M$ domains connected to the backbone, and each domain is denoted as $D_{i}(1 \leq$ $i \leq M)$. Assume that the HA is located in $D_{H}(1 \leq H \leq$ $M)$, the $C N$ is located in $D_{C}(1 \leq C \leq M)$, and $H \neq C$.

The transmission delay assumptions for a single packet are as follows. We assume that packet sizes are equal. (The different packet size case can be analyzed in an analogous manner.) The transmission delay in the backbone between any two domain entries is assumed to be a small value $\delta$, since the transmission delay in the fiber backbone is very low. The transmission delay between $D_{i}$ to its backbone entry is $d_{i}$, and its expectation is $d$. The transmission delay between any two

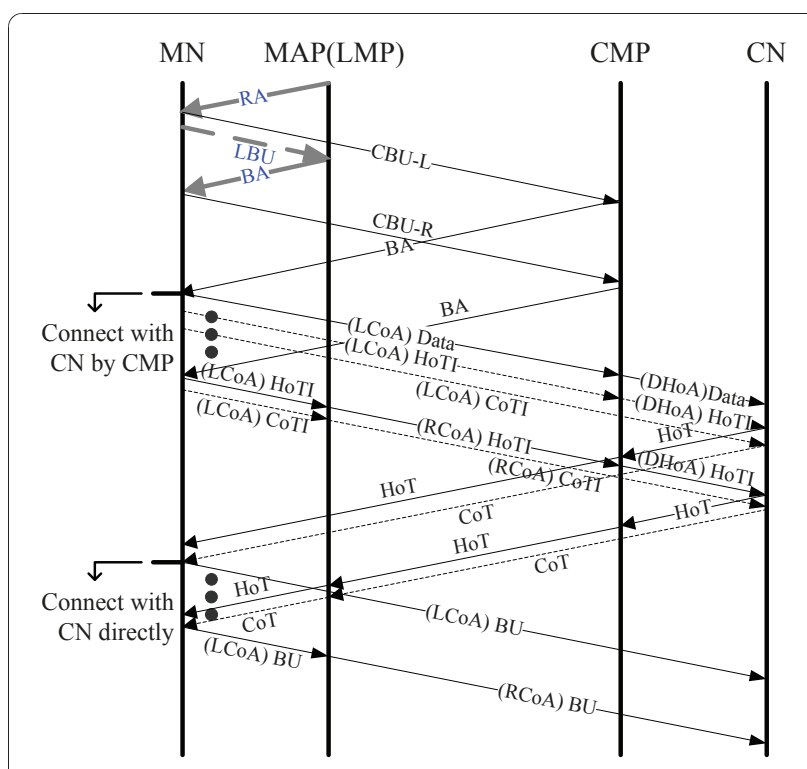

Figure 6 Inter-domain handoff process of ADA in RO mode 


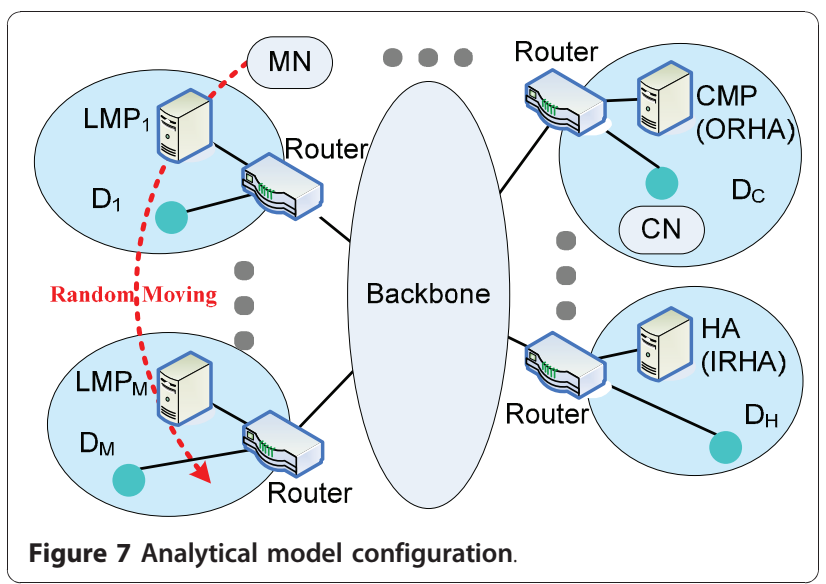

computers in $D_{i}$ is $\Delta_{i}$, and its expectation is $\Delta . d_{i}$ and $\Delta_{i}$ are independent.

The handoff process consists of three phases: (a) handoff detection and triggering; (b) CoA configuration; and (c) binding update. Generally, the combined time of the first two phases is approximately constant and its expectation is denoted as $\rho$ in this article.

Denote the round trip times (RTTs) between $\mathrm{MN}$ and $\mathrm{HA}, \mathrm{HA}$ and $\mathrm{CN}, \mathrm{MN}$, and $\mathrm{CN}$ as $\mathrm{RTT}_{\mathrm{MN}-\mathrm{HA}}, \mathrm{RTT}_{\mathrm{HA}-}$ $C N$, and $\mathrm{RTT}_{M N-C N}$, respectively. Let $\mathrm{RTT}_{M N-H A-C N}$ represent the RTT from $\mathrm{MN}$ to $\mathrm{HA}$, and then to $\mathrm{CN}$. Thus, there will be $\mathrm{RTT}_{\mathrm{MN}-\mathrm{HA}-\mathrm{CN}}=\mathrm{RTT}_{\mathrm{MN}-\mathrm{HA}}+$ RTT $_{\text {HA-CN. }}$.

In addition, we introduce two metrics to reflect the MN's mobility. $\alpha$ is the handoff cycle and we assume that there will be one handoff in an average time period of $\alpha$ seconds. $\beta$ is the indicator of the inter-domain handoff frequency. We assume that there will be one inter-domain handoff in an average number of $\beta+1$ handoffs. As far as applications are concerned, we assume that for a given application, there will be $\gamma$ interactions between the $\mathrm{MN}$ and the $\mathrm{CN}$. We use the total transmission time of the application traffic as one of the evaluation metrics for different mobility protocols. In the performance analysis, we do not consider the processing time in each node.

Next, we derive the handoff latency, single interaction delay between the $\mathrm{MN}$ and the $\mathrm{CN}$, and the total time cost for specific application traffic in BT mode and in RO mode for MIPv6, HMIPv6, CNLP, and ADA. Generally, the handoff latency is defined as the time interval during which an $\mathrm{MN}$ cannot send or receive any packets during handoff. According to this definition, the handoff latency of the RO mode should be the same as that of the BT mode because an $\mathrm{MN}$ will resume communication when it finishes the binding update to its HA. In order to compare performance of these protocols in RO mode, in this section, we define the valid handoff latency in RO mode as the gap between 'the starting point of L2 handoff', and 'the time that the MN has bound its new CoA with the $\mathrm{CN}$ '. In 'Performance evaluation' section, we use the general definition of handoff latency for both BT mode and RO mode in NS2 simulations.

\section{Performance analysis of MIPv6}

If the $\mathrm{MN}$ uses the $\mathrm{BT}$ mode to communicate with the $\mathrm{CN}$, it will only send the BU to the HA. On the other hand, if the MN uses the RO mode, after sending a BU to the $\mathrm{HA}$, it will initiate a RR procedure through the HA. Upon successfully completing the RR procedure, and after receiving a successful BA from the HA, a BU will be sent to the $\mathrm{CN}$.

\section{Bidirectional tunneling mode}

The handoff latency expectation of MIPv6 in BT mode is given by:

$$
\begin{aligned}
& E_{\mathrm{MIPv} 6}^{\mathrm{BT}}(L)=\rho+E\left(\mathrm{RTT}_{\mathrm{MN}-\mathrm{HA}}\right) \\
& =\rho+2 E\left(\Delta_{N}+d_{N}+\delta+d_{H}+\Delta_{H}\right)=4 d+4 \Delta+2 \delta+\rho
\end{aligned}
$$

The delay expectation of a single interaction between the MN and the CN of MIPv6 in BT mode is given by:

$$
E_{\mathrm{MIPv} 6}^{\mathrm{BT}}(D)=E\left(\mathrm{RTT}_{\mathrm{MN}}-\mathrm{HA}-\mathrm{CN}\right)=8 d+8 \Delta+4 \delta
$$

The total time expectation of the traffic with $\gamma$ interactions between the $\mathrm{MN}$ and $\mathrm{CN}$ is the sum of an infinite geometric series. Since $\frac{E_{\mathrm{MIPv}}^{\mathrm{BT}}(L)}{\alpha}<1$, its limit can be given by:

$$
E_{\mathrm{MIPv} 6}^{\mathrm{BT}}(T)=\frac{\gamma \cdot E_{\mathrm{MIPv6}}^{\mathrm{BI}}(D)}{1-\frac{E_{\mathrm{MIPv} 6}^{\mathrm{BT}}(L)}{\alpha}}=\frac{\alpha \cdot \gamma(8 d+8 \Delta+4 \delta)}{\alpha-4 d-4 \Delta-2 \delta-\rho}
$$

The relevant deductions for obtaining Equation 3 are provided in Appendix A.

\section{Route optimization mode}

Assume that every link uses the OSPF (open shortest path first) algorithm to route packets. Thus, in the RR procedure, the transmission delay of HoTI and HoT will be longer than that of CoTI and CoT. The binding update process of MIPv6 in RO mode includes the binding update to the HA, RR procedure, and the binding update to the $\mathrm{CN}$. Without considering packet loss of signaling traffic, the handoff latency expectation of MIPv6 in RO mode is given by:

$$
\begin{aligned}
& E_{\mathrm{MIPv} 6}^{\mathrm{RO}}(L)=\rho+E_{\mathrm{MIPv} 6}^{\mathrm{RO}}(\mathrm{BU}) \\
& =\rho+E\left(\mathrm{RTT}_{\mathrm{MN}}-\mathrm{HA}+\mathrm{RTT}_{\mathrm{MN}}-\mathrm{HA}-\mathrm{CN}+\frac{1}{2} \mathrm{RTT}_{\mathrm{MN}-\mathrm{CN}}\right) \\
& =\rho+E\left(5 d_{N}+6 d_{H}+3 d_{C}+5 \Delta_{N}+6 \Delta_{H}+3 \Delta_{C}+7 \delta\right) \\
& =14 d+14 \Delta+7 \delta+\rho
\end{aligned}
$$


The delay expectation of a single interaction between the $\mathrm{MN}$ and the $\mathrm{CN}$ of MIPv6 in RO mode is given by:

$$
E_{\mathrm{MIPv} 6}^{\mathrm{RO}}(D)=E\left(\mathrm{RTT}_{\mathrm{MN}-\mathrm{CN}}\right)=4 d+4 \Delta+2 \delta
$$

The total time expectation of the traffic with $\gamma$ interactions between the MN and the CN of MIPv6 in RO mode is:

$$
E_{\mathrm{MIPv} 6}^{\mathrm{RO}}(T)=\frac{\gamma \cdot E_{\mathrm{MIPv} 6}^{\mathrm{RO}}(D)}{1-\frac{E_{\mathrm{MIPv} 6}^{\mathrm{RO}}(L)}{\alpha}}=\frac{\alpha \cdot \gamma(4 d+4 \Delta+2 \delta)}{\alpha-14 d-14 \Delta-7 \delta-\rho}
$$

The relevant deductions for obtaining Equation 6 are analogous to the deductions for Equation 3 as shown in Appendix A.

\section{Performance analysis of HMIPv6 \\ Bidirectional tunneling mode}

HMIPv6 classifies handoff into intra-domain handoff and inter-domain handoff. During intra-domain handoff, the MN only sends a LBU to register the new LCoA with its MAP. In this case, the $\mathrm{RCoA}$ remains unchanged. During inter-domain handoff, after registering with the new MAP, the MN must register its new RCoA with its HA.

The expectation of intra-domain handoff latency of HMIPv6 in BT mode is given by:

$$
\begin{aligned}
& E_{\mathrm{HMIPv} 6}^{\mathrm{BT}-\operatorname{INTA}^{2}}(L)=\rho+E\left(\mathrm{RTT}_{\mathrm{MN}-\mathrm{MAP}}\right) \\
& =\rho+2 E\left(\Delta_{N}\right)=2 \Delta+\rho
\end{aligned}
$$

The expectation of inter-domain handoff latency of HMIPv6 in BT mode is given by:

$$
\begin{aligned}
& E_{\mathrm{HMIPv6}}^{\mathrm{BT}-\mathrm{INTER}}(L) \\
& =\rho+E\left(\mathrm{RTT}_{\mathrm{MN}}-\mathrm{MAP}\right)+E\left(\mathrm{RTT}_{\mathrm{MN}}-\mathrm{MAP}-\mathrm{HA}\right) \\
& =4 d+8 \Delta+2 \delta+\rho
\end{aligned}
$$

The delay expectation of a single interaction between the $\mathrm{MN}$ and the $\mathrm{CN}$ of HMIPv6 in BT mode is given by:

$$
E_{\mathrm{HMIPv} 6}^{\mathrm{BT}}(D)=E\left(\mathrm{RTT}_{\mathrm{MN}}-\mathrm{MAP}-\mathrm{HA}-\mathrm{CN}\right)=8 d+10 \Delta+4 \delta
$$

The total time expectation of the traffic with $\gamma$ interactions between the MN and the CN of HMIPv6 in BT mode is:

$$
\begin{aligned}
& E_{\mathrm{HMIPv} 6}^{\mathrm{BT}}(T) \\
& =\frac{\gamma \cdot E_{\mathrm{HMIPv} 6}^{\mathrm{BT}}(D)}{1-\frac{E_{\mathrm{HMIPv} 6}^{\mathrm{BT}-\mathrm{INTER}^{2}}(L)+\beta \cdot E_{\mathrm{HMIPv}}^{\mathrm{BT}-\mathrm{INTRA}^{2}}(L)}{\alpha(\beta+1)}} \\
& =\frac{\alpha \gamma(\beta+1)(8 d+10 \Delta+4 \delta)}{\alpha \beta+\alpha-2 \beta \Delta-\beta \rho-4 d-8 \Delta-2 \delta-\rho}
\end{aligned}
$$

The relevant deductions for obtaining Equation 10 are provided in Appendix B.

Route optimization mode

The expectation of intra-domain handoff latency of HMIPv6 in RO mode is given by:

$$
E_{\mathrm{HMIPv} 6}^{\mathrm{RO}-\operatorname{INTRA}}(L)=\rho+E\left(\mathrm{RTT}_{\mathrm{MN}}-\mathrm{MAP}\right)=2 \Delta+\rho
$$

The expectation of inter-domain handoff latency of HMIPv6 in RO mode is given by:

$$
\begin{aligned}
& E_{\mathrm{HMIPv}}^{\mathrm{RO}-\text { INTRR }}(L)=\rho+E\left(\mathrm{RTT}_{\mathrm{MN}}-\mathrm{MAP}\right)+E_{\mathrm{HMIPv}}^{\mathrm{RO}-\mathrm{INTER}}(B U) \\
& =\rho+E\left(\mathrm{RTT}_{\mathrm{MN}}-\mathrm{MAP}\right)+E\left(\mathrm{RTT}_{\mathrm{MN}}-\mathrm{MAP}-\mathrm{HA}\right. \\
& \left.+\mathrm{RTT}_{\mathrm{MN}}-\mathrm{MAP}-\mathrm{HA}-\mathrm{CN}+\frac{1}{2} \mathrm{RTT}_{\mathrm{MN}}-\mathrm{MAP}-\mathrm{CN}\right) \\
& =\rho+2 E\left(\Delta_{N}\right) \\
& +E\left(5 d_{N}+6 d_{H}+3 d_{C}+10 \Delta_{N}+6 \Delta_{H}+3 \Delta_{C}+7 \delta\right) \\
& =14 d+21 \Delta+7 \delta+\rho
\end{aligned}
$$

The delay expectation of a single interaction between the MN and the CN of HMIPv6 in RO mode is given by:

$$
E_{\mathrm{HMIPv} 6}^{\mathrm{RO}}(D)=E\left(\mathrm{RTT}_{\mathrm{MN}-\mathrm{MAP}-\mathrm{CN}}\right)=4 d+6 \Delta+2 \delta
$$

The total time expectation of the traffic with $\gamma$ interactions between the $\mathrm{MN}$ and the $\mathrm{CN}$ of HMIPv6 in $\mathrm{RO}$ mode is:

$$
\begin{aligned}
& E_{\mathrm{HMIPv6}}^{\mathrm{RO}}(T) \\
& =\frac{\gamma \cdot E_{\mathrm{HMIPv} 6}^{\mathrm{RO}}(D)}{1-\frac{E_{\mathrm{HMIPv6}}^{\mathrm{RO}-\mathrm{INTER}^{2}}(L)+\beta \cdot E_{\mathrm{HMIPv}}^{\mathrm{RO}-\mathrm{INTRA}}(L)}{\alpha(\beta+1)}} \\
& =\frac{\alpha \gamma(\beta+1)(4 d+6 \Delta+2 \delta)}{\alpha \beta+\alpha-2 \beta \Delta-\beta \rho-14 d-21 \Delta-7 \delta-\rho}
\end{aligned}
$$

The relevant deductions for obtaining Equation 14 are analogous to the deductions for Equation 10 as shown in Appendix B.

\section{Performance analysis of CNLP}

In the analysis of CNLP, we assume that the ORHA is located within the CN's domain and we only consider mobile node-initiated sessions.

\section{Bidirectional tunneling mode}

The handoff latency expectation of CNLP in BT mode is:

$$
E_{\mathrm{CNLP}}^{\mathrm{BT}}(L)=\rho+E\left(\mathrm{RTT}_{\mathrm{MN}} \text { - ORHA }\right)=4 d+4 \Delta+2 \delta+\rho
$$

The delay expectation of a single interaction between the $\mathrm{MN}$ and the CN of CNLP in BT mode is:

$$
E_{\mathrm{CNLP}}^{\mathrm{BT}}(D)=E\left(\mathrm{RTT}_{\mathrm{MN}} \text { - ORHA - CN }\right)=4 d+6 \Delta+2 \delta
$$


In CNLP, the MN needs to run an ORHA discovery procedure before communicating with a new $\mathrm{CN}$, thus the total time expectation of the traffic with $\gamma$ interactions between the $\mathrm{MN}$ and the $\mathrm{CN}$ of CNLP in $\mathrm{BT}$ mode is:

$$
\begin{gathered}
E_{\mathrm{CNLP}}^{\mathrm{BT}}(T)=\frac{\gamma \cdot E_{\mathrm{CNLP}}^{\mathrm{BT}}(D)+2 E\left(\mathrm{RTT}_{\mathrm{MN}}-\mathrm{ORHA}\right)}{1-\frac{E_{\mathrm{CNLP}}^{\mathrm{BT}}(L)}{\alpha}} \\
=\frac{\alpha(\gamma(4 d+6 \Delta+2 \delta)+8 d+8 \Delta+4 \delta)}{\alpha-4 d-4 \Delta-2 \delta-\rho}
\end{gathered}
$$

The relevant deductions for obtaining Equation 17 are analogous to the deductions for Equation 3 as shown in Appendix A.

\section{Route optimization mode}

The handoff latency expectation of CNLP in RO mode is:

$$
\begin{aligned}
& E_{\mathrm{CNLP}}^{\mathrm{RO}}(L)=\rho+E_{\mathrm{CNLP}}^{\mathrm{RO}}(B U) \\
& =\rho+E\left(\mathrm{RTT}_{\mathrm{MN}}-\mathrm{ORHA}+\mathrm{RTT}_{\mathrm{MN}}-\mathrm{ORHA}-\mathrm{CN}+\frac{1}{2} \mathrm{RTT}_{\mathrm{MN}-\mathrm{CN}}\right) \\
& =\rho+E\left(5 d_{N}+5 d_{C}+5 \Delta_{N}+7 \Delta_{C}+5 \delta\right) \\
& =10 d+12 \Delta+5 \delta+\rho
\end{aligned}
$$

The delay expectation of a single interaction between the MN and the CN of CNLP in RO mode is:

$$
E_{\mathrm{CNLP}}^{\mathrm{RO}}(D)=E\left(\mathrm{RTT}_{\mathrm{MN}-\mathrm{CN}}\right)=4 d+4 \Delta+2 \delta
$$

The total time expectation of the traffic with $\gamma$ interactions between the MN and the CN of CNLP in RO mode is:

$$
\begin{aligned}
& E_{\mathrm{CNLP}}^{\mathrm{RO}}(T)=\frac{\gamma \cdot E_{\mathrm{CNLP}}^{\mathrm{RO}}(D)+2 E\left(\mathrm{RTT}_{\mathrm{MN}-\mathrm{ORHA}}\right)}{1-\frac{E_{\mathrm{CNLP}}^{\mathrm{RO}}(L)}{\alpha}} \\
& =\frac{\alpha(\gamma+2)(4 d+4 \Delta+2 \delta)}{\alpha-10 d-12 \Delta-5 \delta-\rho}
\end{aligned}
$$

The relevant deductions for obtaining Equation 20 are analogous to the deductions for Equation 3 as shown in Appendix A.

\section{Performance analysis of ADA}

\section{Bidirectional tunneling mode}

The expectation of intra-domain handoff latency of ADA in BT mode is given by:

$$
\begin{aligned}
& E_{\mathrm{ADA}}^{\mathrm{BT}-\mathrm{INTRA}}(L)=\rho+E\left(\mathrm{RTT}_{\mathrm{MN}-\mathrm{MAP}}\right) \\
& =\rho+2 E\left(\Delta_{N}\right)=2 \Delta+\rho
\end{aligned}
$$

The expectation of inter-domain handoff latency of ADA in $\mathrm{BT}$ mode is given by:

$$
\begin{aligned}
& E_{\mathrm{ADA}}^{\mathrm{BT}-\operatorname{INTER}}(L) \\
& =\rho+E\left(\mathrm{RTT}_{\mathrm{MN}}-\mathrm{CMP}\right)=4 d+4 \Delta+2 \delta+\rho
\end{aligned}
$$

The delay expectation of a single interaction between the $\mathrm{MN}$ and the $\mathrm{CN}$ of $\mathrm{ADA}$ in $\mathrm{BT}$ mode is given by:

$$
E_{\mathrm{ADA}}^{\mathrm{BT}}(D)=E\left(\mathrm{RTT}_{\mathrm{MN}}-\mathrm{CMP}-\mathrm{CN}\right)=4 d+6 \Delta+2 \delta
$$

The total time expectation of the traffic with $\gamma$ interactions between the MN and the $\mathrm{CN}$ of ADA in BT mode is given by:

$$
\begin{aligned}
& E_{\mathrm{ADA}}^{\mathrm{BT}}(T) \\
& =\frac{\gamma \cdot E_{\mathrm{ADA}}^{\mathrm{BT}}(D)+2 E\left(\mathrm{RTT}_{\mathrm{MN}-\mathrm{CMP}}\right)}{1-\frac{E_{\mathrm{ADA}}^{\mathrm{B}-\mathrm{INTER}}(L)+\beta \cdot E_{\mathrm{ADA}}^{\mathrm{BN}-\mathrm{INTRA}}(L)}{\alpha(\beta+1)}} \\
& =\frac{\alpha(\beta+1)(\gamma(4 d+6 \Delta+2 \delta)+8 d+8 \Delta+4 \delta)}{\alpha \beta+\alpha-2 \beta \Delta-\beta \rho-4 d-4 \Delta-2 \delta-\rho}
\end{aligned}
$$

The relevant deductions for obtaining Equation 24 are analogous to the deductions for Equation 10 as shown in Appendix B.

\section{Route optimization mode}

The expectation of intra-domain handoff latency of $\mathrm{ADA}$ in $\mathrm{RO}$ mode is given by:

$$
\begin{aligned}
& E_{\mathrm{ADA}}^{\mathrm{RO}-{ }^{-I T R A}}(L) \\
& =\rho+E\left(\mathrm{RTT}_{\mathrm{MN}}-\mathrm{MAP}\right)+E\left(\mathrm{RTT}_{\mathrm{MN}}-\mathrm{MAP}-\mathrm{CN}\right)+\frac{1}{2} E\left(\mathrm{RTT}_{\mathrm{MN}}-\mathrm{CN}\right) \\
& =6 d+10 \Delta+3 \delta+\rho
\end{aligned}
$$

The expectation of inter-domain handoff latency of ADA in $\mathrm{RO}$ mode is given by:

$$
\begin{aligned}
& E_{\mathrm{ADA}}^{\mathrm{RO}-\operatorname{INTER}}(L) \\
& =\rho+E\left(\mathrm{RTT}_{\mathrm{MN}}-\mathrm{CMP}\right)+E\left(\mathrm{RTT}_{\mathrm{MN}}-\mathrm{CMP}-\mathrm{CN}\right)+\frac{1}{2} E\left(\mathrm{RTT}_{\mathrm{MN}-\mathrm{CN}}\right) \\
& =10 d+12 \Delta+5 \delta+\rho
\end{aligned}
$$

The delay expectation of a single interaction between the $\mathrm{MN}$ and the $\mathrm{CN}$ of ADA in RO mode is given by:

$$
E_{\mathrm{ADA}}^{\mathrm{RO}}(D)=E\left(\mathrm{RTT}_{\mathrm{MN}-\mathrm{CN}}\right)=4 d+4 \Delta+2 \delta
$$

The total time expectation of the traffic with $\gamma$ interactions between the $\mathrm{MN}$ and the $\mathrm{CN}$ of $\mathrm{ADA}$ in RO mode is given by:

$$
\begin{aligned}
& E_{\mathrm{ADA} A}^{\mathrm{RO}}(T) \\
& =\frac{\gamma \cdot E_{\mathrm{ADA}}^{\mathrm{RO}}(D)+2 E\left(\mathrm{RTT}_{\mathrm{MN}}-\mathrm{CMP}\right)}{1-\frac{E_{\mathrm{ADA}}^{\mathrm{OO}}-\mathrm{INTER}^{2}(L)+\beta \cdot E_{\mathrm{ADA}}^{\mathrm{RO}}-\mathrm{NTRA}(L)}{\alpha(\beta+1)}} \\
& =\frac{\alpha(\gamma+2)(\beta+1)(4 d+4 \Delta+2 \delta)}{\alpha \beta+\alpha-6 \beta d-10 \beta \Delta-3 \beta \delta-\beta \rho-10 d-12 \Delta-5 \delta-\rho}
\end{aligned}
$$

The relevant deductions for obtaining Equation 28 are analogous to the deductions for Equation 10 as shown in Appendix B. 


\section{Performance evaluation Numerical results}

Using the analytical framework proposed in 'Performance analysis' section, we evaluate and compare performance of MIPv6, HMIPv6, CNLP, and ADA by numerical analysis. The numerical analysis parameters are shown in Table 1.

Figure 8 shows the change trend of the total transmission time $E(T)$ of the traffic with $\gamma$ interactions between the $\mathrm{MN}$ and $\mathrm{CN}$, where the handoff cycle is $\alpha$. The values of $\gamma$ range from 100 to 300 and increase in steps of 50, while the values of $\alpha$ range from 10 to $40 \mathrm{~s}$ and increase in steps of $10 \mathrm{~s}$. In Figure 8, the four planes from top to bottom indicate $E(T)$ of MIPv6, HMIPv6, CNLP and ADA, respectively.

As can be seen from Figure 8, $E(T)$ of ADA is slightly shorter than that of CNLP and significantly shorter than that of MIPv6 and HMIPv6. More specifically, the average total transmission time for ADA is $49.9 \%$ shorter than for MIPv6, 49.0\% shorter than for HMIPv6 and $3.3 \%$ shorter than for CNLP.

Figure 9 shows the change trend of the total handoff latency (THL) as a function of $\gamma$ and $\alpha$. THL indicates the break-off time of an application caused by handoff and directly influences user perceived QoS.

As can be seen from Figure 9, the average THL for ADA is $64.8 \%$ shorter than for MIPv6, 49.4\% shorter than for HMIPv6 and $32.1 \%$ shorter than for CNLP. Between the other three protocols, THL for HMIPv6 is markedly shorter than for MIPv6. When $\gamma=300$ and $\alpha$ $=10 \mathrm{~s}$, THL for HMIPv6 is only $67.2 \%$ of that for MIPv6. THL for CNLP is shorter than that for HMIPv6. In fact, although the single time handoff latency for HMIPv6 is shorter than that for CNLP, the total handoff latency for CNLP is much shorter because its shorter total transmission time will experience fewer handoffs.

\section{Simulation results}

We have transferred the NS2 MobiWan [29] extension for MIPv6 to ns-2.31 [30] and implemented in it additional functions such as establishment of bidirectional tunneling, the return routability procedure, and support for the retransmission of BUs. We have also completely implemented HMIPv6, CNLP, and ADA in NS2. The simulation topology is the same as that used for the numerical analysis in Figure 7. We take four wireless domains $\left(D_{1}-D_{4}\right)$ and one wired domain $\left(D_{5}\right)$ to connect to the backbone. The HA/IRHA is located in $D_{1}$, and the CMP/ORHA and $\mathrm{CN}$ are located in $D_{5}$.

As far as the MAP is concerned, we have simulated two extreme cases. In case 1 , all wireless domains belong to one MAP domain, which means that every handoff is an intra-domain handoff. In case 2, there is one MAP in each wireless domain and every handoff will be an inter-domain handoff.

The MN takes a random rectilinear motion without pause between four wireless domains $\left(D_{1}-D_{4}\right)$ at a speed of $2 \mathrm{~m} / \mathrm{s}$, and communicates with the $\mathrm{CN}$ located in $D_{5}$ to download data by FTP. Each scenario is simulated for $10000 \mathrm{~s}$ and the results are shown in Figures 10, 11, 12 and 13 and Tables 2 and 3 .

Figures 10 and 11 show segments of the TCP traces for MIPv6, HMIPv6, CNLP, and ADA during one handoff in $\mathrm{BT}$ mode and $\mathrm{RO}$ mode, respectively. The $x$-axis is the time (s) and the $y$-axis is the number of FTP packets received by the MN in one second. We choose the segment of $50 \mathrm{~s}$ (from $5 \mathrm{~s}$ before the handoff to $45 \mathrm{~s}$ after the handoff) to compare the protocol performance.

As can be seen from Figure 10, when there is no handoff, the TCP throughput of ADA and CNLP in BT mode is close to each other and significantly better than that of MIPv6 and HMIPv6. When the MN experiences one handoff, there will be a service disruption in TCP for all four protocols. However, the TCP throughput of ADA recovers fastest in these four protocols. During the $15 \mathrm{~s}$ after the intra-domain handoff, ADA will on average receive $3.87,9.1$, and 9.3 more packets every second than CNLP, HMIPv6, and MIPv6. During the $15 \mathrm{~s}$ after the inter-domain handoff, ADA and CNLP will on average receive 5.47 more packets every second than HMIPv6 and MIPv6. Actually, the intra-domain handoff latency for HMIPv6 is shorter than for CNLP and for MIPv6. However, the TCP throughput for CNLP is higher than for HMIPv6 in this case because of its shorter packet transmission delay in BT mode.

Table 1 Numerical analysis parameters.

\begin{tabular}{|c|c|c|}
\hline Parameters & Symbols & Values \\
\hline Handoff cycle & $\alpha$ & $10-40 \mathrm{~s}$ \\
\hline Inter-domain handoff frequency & $\beta$ & 10 \\
\hline Time expectation of handoff detection and CoA configuration & $\rho$ & $1300 \mathrm{~ms}$ \\
\hline Transmission delay expectation between one domain to its backbone entry & $d$ & $150 \mathrm{~ms}$ \\
\hline Transmission delay expectation between any two computers in one domain & $\Delta$ & $10 \mathrm{~ms}$ \\
\hline Transmission delay in backbone between any two domain entries & $\delta$ & $5 \mathrm{~ms}$ \\
\hline Expected interactions between $\mathrm{MN}$ and $\mathrm{CN}$ & $\gamma$ & $100-300$ \\
\hline
\end{tabular}




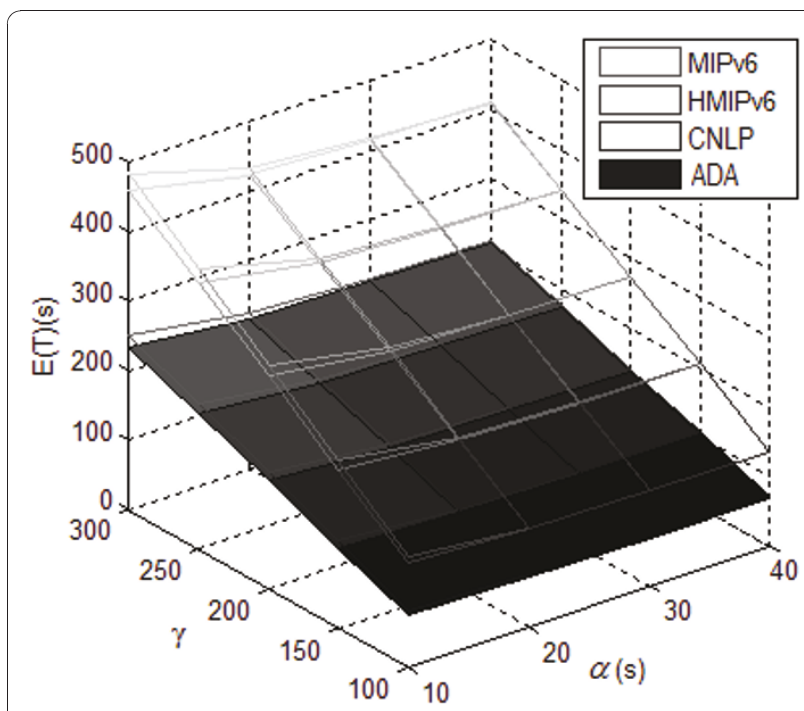

Figure 8 Total transmission time of MIPv6, HMIPv6, CNLP, and ADA in BT mode as a function of $\gamma$ and $\alpha$.

As shown in Figure 11, when there is no handoff, the TCP throughputs of all four protocols in RO mode are close to each other. When there is an intra-domain handoff, the performance of ADA is close to HMIPv6 and better than CNLP and MIPv6. When there is an inter-domain handoff, the performance of ADA is close to CNLP and better than HMIPv6 and MIPv6.

In order to make further investigation to the data interaction process during handoff, we calculate the transmission delay (TD) of every packet in TCP-sink and show the results in Figures 12 and 13. The $x$-axis is

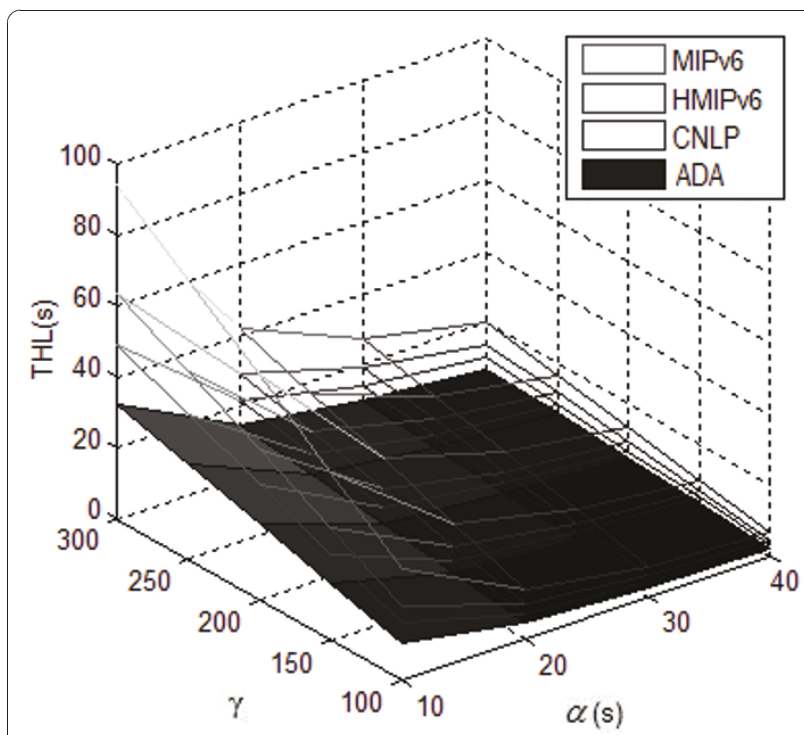

Figure 9 Total handoff latency of MIPv6, HMIPv6, CNLP, and ADA in BT mode as a function of $\gamma$ and $\alpha$. the receiving time (s) of the packet and the $y$-axis is the transmission delay of the packet (ms).

As can be seen from Figures 12 and 13, there are obvious assembly effects in packet transmission delay. For example, the TD for ADA in BT mode is generally $406 \mathrm{~ms}$. However, after handoff, there will be some packets with TD of 413 or $415 \mathrm{~ms}$. If we follow the trace of one packet, we will find that this phenomenon occurs because the TCP-source may send two packets simultaneously and this results in queuing in the transmission link. In this case, the following packet will arrive about 7 or $9 \mathrm{~ms}$ later than the previous one.

In Figure 12, we compare the packet transmission delay for CNLP and ADA in BT mode. Performance of HMIPv6 and MIPv6 in BT mode is significantly worse than that of these two protocols and is therefore omitted in this case. As shown in Figure 12, TD of ADA and CNLP in BT mode is only about $406 \mathrm{~ms}$ when there is no handoff. After the handoff, there will be some packets experiencing higher transmission delay. As far as the break-off time of TCP is concerned, ADA breaks $2.54 \mathrm{~s}$ in intra-domain handoff and $5.88 \mathrm{~s}$ in inter-domain handoff; while CNLP breaks $5.89 \mathrm{~s}$ for both types of handoffs. As a result, ADA will recover faster from intra-domain handoff than CNLP.

As shown in Figure 13, the packet transmission delay for CNLP and ADA in RO mode is shorter than in BT mode. During intra-domain handoff, the break-off time for ADA and HMIPv6 is short, about 2.42 and $2.53 \mathrm{~s}$, respectively; while the break-off time of CNLP in this case is about $5.60 \mathrm{~s}$. During inter-domain handoff, the break-off time for ADA and CNLP is about 5.62 and $5.60 \mathrm{~s}$, respectively; while the break-off time of HMIPv6 in this case is about $6.89 \mathrm{~s}$. As a result, there is a service interruption in HMIPv6 and the MN needs to reset its TCP connection instead of making fast retransmission as in other protocols. During the interruption in HMIPv6, only three packets are forwarded by the HA with a transmission delay of $1413 \mathrm{~ms}$.

In Tables 2 and 3, the average TCP throughput, average packet transmission delay, and average handoff latency are calculated for MIPv6, HMIPv6, CNLP, and ADA. In addition, the number of signaling packets for one handoff has also been calculated to compare the average handoff cost of these four protocols.

As shown in Tables 2 and 3, the signaling cost of ADA will be relatively higher than the other three protocols. As shown in Table 2, in BT mode, ADA increases by 2 to 4 packets the signaling cost for one handoff compared to MIPv6 and CNLP. However, ADA will achieve $418 \%$ higher TCP throughput, $70.7 \%$ shorter transmission delay, and $96.6 \%$ shorter handoff latency than MIPv6 in intra-domain handoff. It also can achieve $3.8 \%$ higher TCP throughput and $93.2 \%$ shorter handoff 


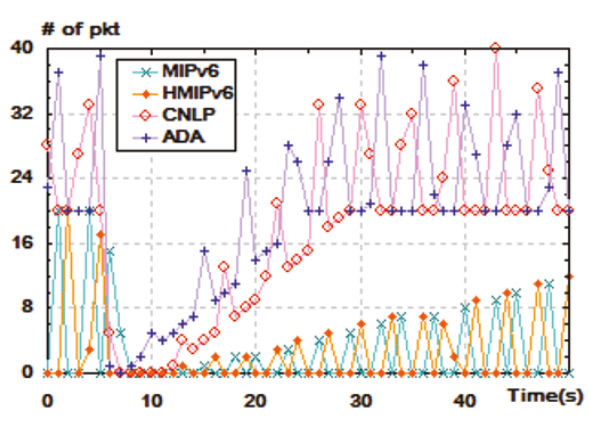

(a)

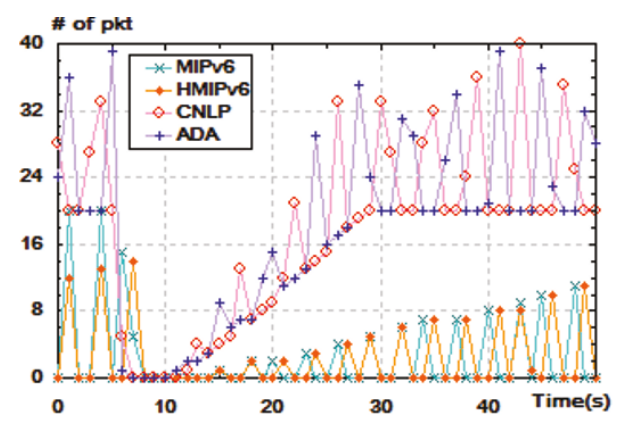

(b)

Figure 10 TCP traces for MIPv6, HMIPv6, CNLP, and ADA in BT mode. (a) Intra-domain handoff; (b) inter-domain handoff.

latency than CNLP in this case. When compared with HMIPv6, although ADA increases the signaling cost by two packets, it will achieve $275 \%$ higher TCP throughput, $71.1 \%$ shorter transmission delay, and $1.8 \%$ shorter handoff latency in intra-domain handoff; 636\% higher TCP throughput, $71.1 \%$ shorter transmission delay, and $53.0 \%$ shorter handoff latency in inter-domain handoff. Actually, the total signaling cost of ADA in the entire NS2 simulation is only 6.25 to $9.38 \mathrm{kB}$ for 200 handoffs in BT mode and 15.6 to $18.8 \mathrm{kB}$ for 200 handoffs in RO mode. Such small signaling traffic can be ignored in most network environments.

What should be noted is that in the NS2 simulation topology, there is only one CMP/ORHA. In fact, the signaling cost of ADA and CNLP will increase as the number of CMP/ORHA increases. However, the number of $\mathrm{CNs}$ that are communicating with one $\mathrm{MN}$ at the same time and whose applications all need mobile IP support is very limited. Thus, the number of CMP/ORHA that an MN holds simultaneously is small. Since these
CMPs/ORHAs are located in different network domains, the signaling packets will be sent to different destinations, thereby further reducing the possibility of congestion. More research will be conducted to implement further system simplicity in the real system deployment.

\section{Conclusions}

In this article, we have proposed ADA, a distributed mobile IP-compatible mobility management architecture. In $\mathrm{ADA}$, there are two asymmetric mobility agents to serve each end-to-end communication. One mobility agent is located close to the $\mathrm{MN}$ and the other is located close to the CN. We apply ADA to MIPv6 communications and present the detailed protocol design. ADA can significantly reduce handoff latency and provide an efficient route for all $\mathrm{CNs}$ including those that do not support route optimization. It also eliminates congestion at the MN's HA and home link, and reduces the impact of any possible failures of the HA and home link on the path to or from the MN. In addition, it

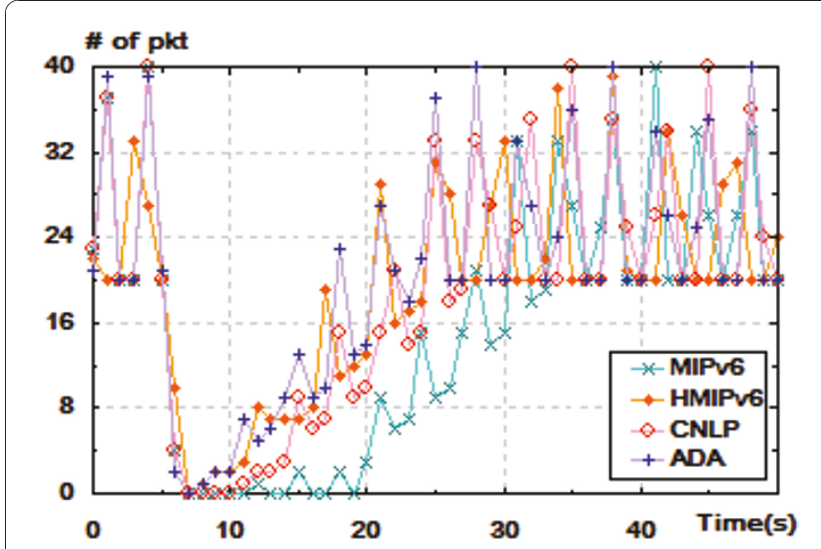

(a)

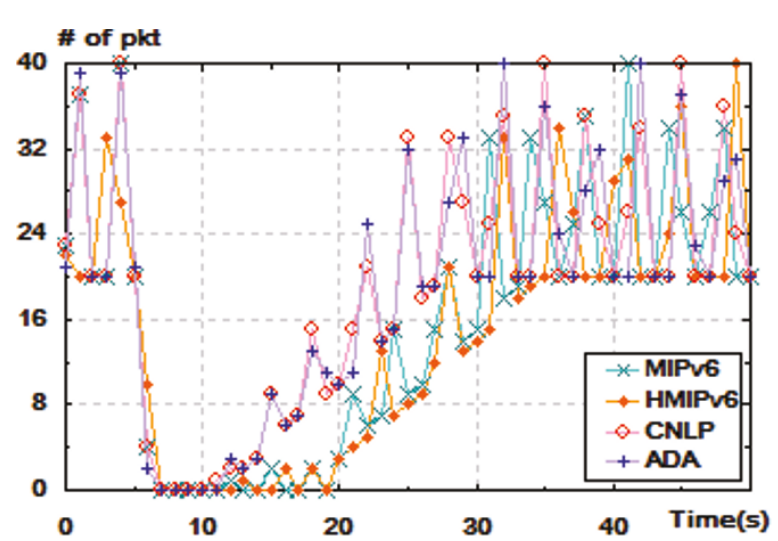

(b)

Figure 11 TCP traces for MIPv6, HMIPv6, CNLP, and ADA in RO mode. (a) Intra-domain handoff; (b) inter-domain handoff. 


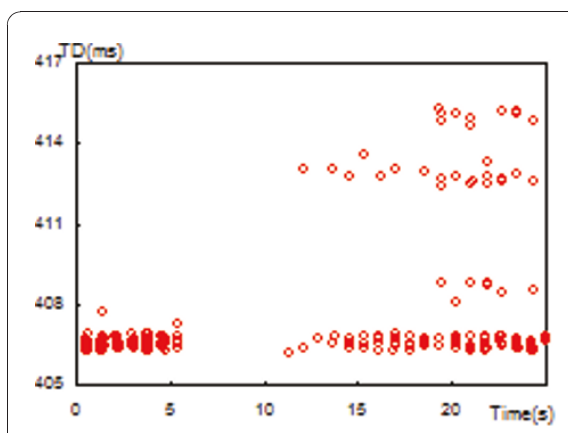

(a)

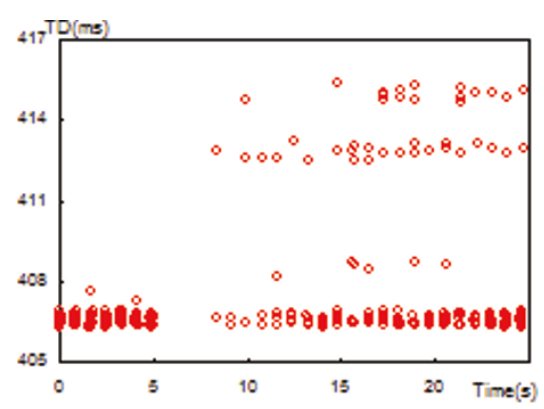

(b)

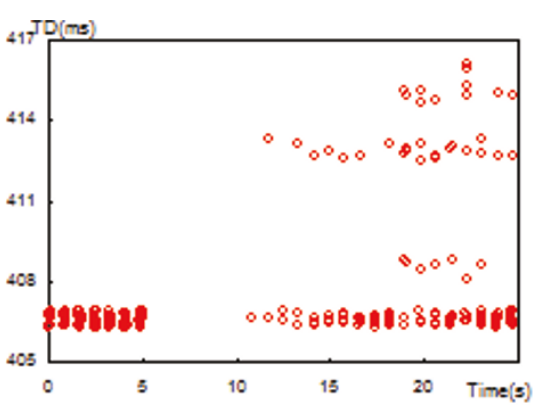

(c)

Figure 12 Packet transmission delay for CNLP and ADA in BT mode. (a) CNLP; (b) ADA-Intra; (c) ADA-Inter.

provides an easy-to-use mechanism for MNs to manage and control each traffic session with a different policy based on practical requirements. Generally, sessions that need to be maintained during the MN's mobility are mainly client-server types where $\mathrm{CNs}$ act as servers. ADA is well-suited for this case and can help such CNs enhance their support for their clients' mobility without any change to the CN's implementation. It is also convenient for the $\mathrm{CN}$-located network to monitor and control in-bound and out-bound traffic and provide specific QoS support. ADA does not require modifications at $\mathrm{CNs}$ and HAs, but only moderate modifications at MNs. It is also backward compatible with Mobile IP and can be incrementally deployed. Numerical and simulation results both indicate that ADA achieves better performance than MIPv6, HMIPv6 and CNLP.

\section{Appendix A}

The relevant deductions for obtaining Equation 3 are given below. The relevant deductions for Equations 6, 17 , and 20 can be obtained in an analogous way.

Assume the interaction number for a given application is $\gamma$. Thus the transmission time of the data packets for MIPv6 in BT mode should be $\gamma \cdot E_{\mathrm{MIPv} 6}^{\mathrm{BT}}(D)$. Based on the definition of $\alpha$, there will be $\gamma \cdot E_{\mathrm{MIPv} 6}^{\mathrm{BT}}(D) / \alpha$ of handoffs during this period. As a result, the total

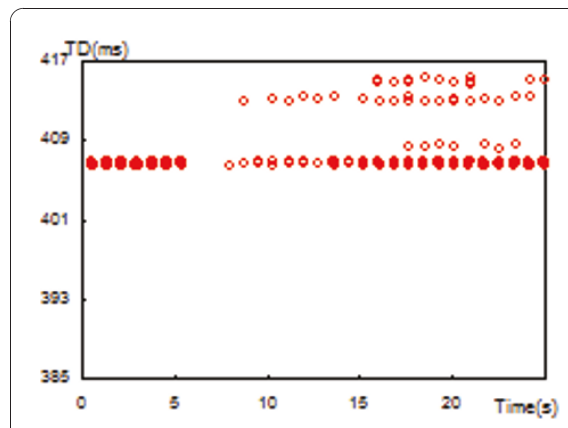

(a)

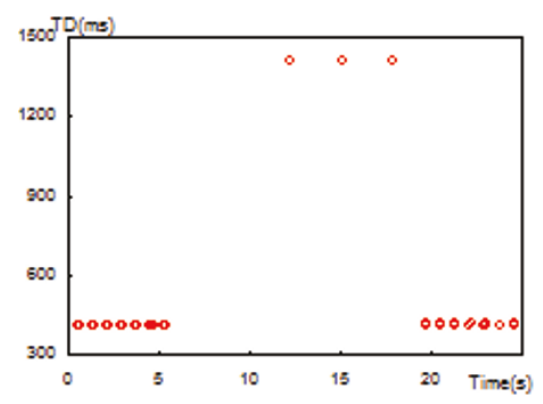

(d)

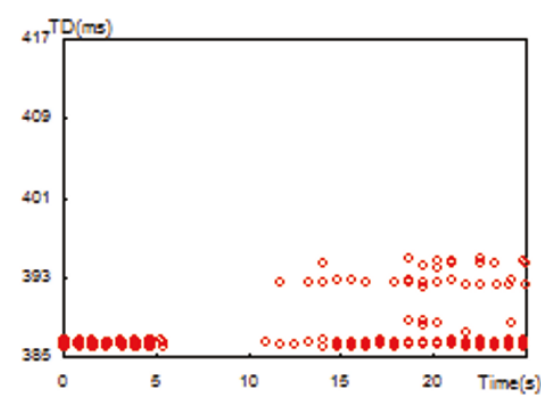

(b)

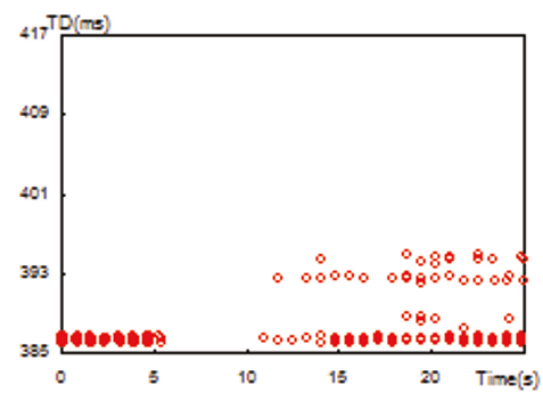

(e)

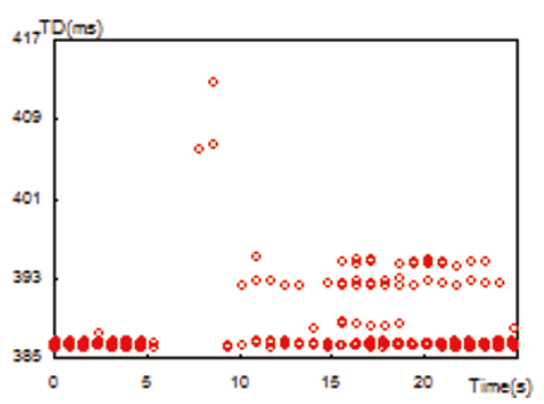

(c)

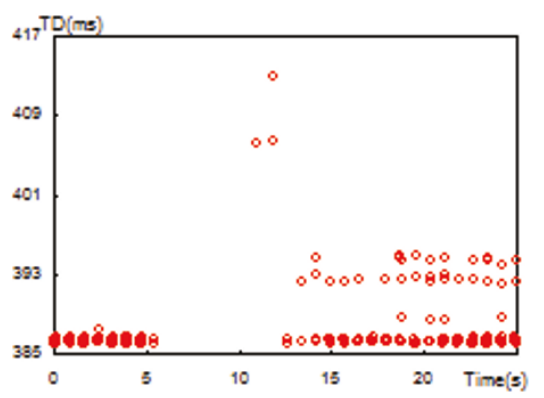

(f)

Figure 13 Packet transmission delay for HMIPv6, CNLP, and ADA in RO mode. (a) HMIPv6-Intra; (b) CNLP-Intra; (c) ADA-Intra; (d) HMIPv6Inter; (e) CNLP-Inter; (f) ADA-Inter. 
Table 2 The ns2 simulation results of MIPv6, HMIPv6, CNLP and ADA in BT mode.

\begin{tabular}{lllll}
\hline Type & $\begin{array}{l}\text { TCP } \\
\text { throughput } \\
\text { (kbps) }\end{array}$ & $\begin{array}{l}\text { Transmission } \\
\text { delay of } \mathbf{1} \text { pkt } \\
\text { (ms) }\end{array}$ & $\begin{array}{l}\text { Handoff } \\
\text { latency } \\
\text { (ms) }\end{array}$ & $\begin{array}{l}\text { Handoff } \\
\text { cost (pkt) }\end{array}$ \\
\hline MIPv6 & 45.4 & 1389 & 1621 & 2 \\
$\begin{array}{l}\text { HMIPv6- } \\
\text { Intra }\end{array}$ & 62.6 & 1408 & 56 & 2 \\
$\begin{array}{l}\text { HMIPv6- } \\
\text { Inter }\end{array}$ & 30.8 & 1409 & 1705 & 4 \\
$\begin{array}{l}\text { CNLP } \\
\text { ADA- }\end{array}$ & 226.5 & 407 & 809 & 2 \\
Intra & 235.0 & 407 & 55 & 4 \\
$\begin{array}{l}\text { ADA- } \\
\text { Inter }\end{array}$ & 226.8 & 407 & 801 & 6 \\
\hline
\end{tabular}

transmission time of this traffic for MIPv6 will be increased by $\left(\gamma \cdot E_{\mathrm{MIPv} 6}^{\mathrm{BT}}(D) / \alpha\right) \cdot E_{\mathrm{MIPv} 6}^{\mathrm{BT}}(L)$. Of course, during the increased time, we also need to consider the increased handoff and the new time cost brought by the increased handoff. This is given by a standard infinite geometric series and its sum should be:

$$
\begin{aligned}
E_{\mathrm{MIPv6}}^{\mathrm{BT}}(T) & =\gamma \cdot E_{\mathrm{MIPv} 6}^{\mathrm{BT}}(D)+\gamma \cdot E_{\mathrm{MIPv} 6}^{\mathrm{BT}}(D) \frac{E_{\mathrm{MIPv} 6}^{\mathrm{BT}}(L)}{\alpha} \\
& +\gamma \cdot E_{\mathrm{MIPv} 6}^{\mathrm{BT}}(D)\left(\frac{E_{\mathrm{MIPv} 6}^{\mathrm{BT}}(L)}{\alpha}\right)^{2}+\ldots
\end{aligned}
$$

The common ratio in this infinite geometric series is $q=\frac{E_{\mathrm{MIPv} 6}^{\mathrm{BT}}(L)}{\alpha}<1$. Thus from the finite sum formulae, we can get Equation 3:

$$
E_{\mathrm{MIPv} 6}^{\mathrm{BT}}(T)=\frac{\gamma \cdot E_{\mathrm{MIPv} 6}^{\mathrm{BT}}(D)}{1-\frac{E_{\mathrm{MIPv}}^{\mathrm{BT}}(L)}{\alpha}}=\frac{\alpha \cdot \gamma(8 d+8 \Delta+4 \delta)}{\alpha-4 d-4 \Delta-2 \delta-\rho}
$$

Table 3 The ns2 simulation results of MIPv6, HMIPv6, CNLP and ADA in RO mode.

\begin{tabular}{lllll}
\hline Type & $\begin{array}{l}\text { TCP } \\
\text { throughput } \\
\text { (kbps) }\end{array}$ & $\begin{array}{l}\text { Transmission } \\
\text { delay of 1 pkt } \\
\text { (ms) }\end{array}$ & $\begin{array}{l}\text { Handoff } \\
\text { latency } \\
\text { (ms) }\end{array}$ & $\begin{array}{l}\text { Handoff } \\
\text { cost (pkt) }\end{array}$ \\
\hline MIPv6 & 187.5 & 394 & 1621 & 8 \\
$\begin{array}{l}\text { HMIPv6- } \\
\text { Intra }\end{array}$ & 237.2 & 407 & 57 & 8 \\
$\begin{array}{l}\text { HMIPv6- } \\
\text { Inter }\end{array}$ & 176.2 & 414 & 1705 & 10 \\
CNLP & 239.3 & 387 & 797 & 8 \\
ADA- & 249.6 & 387 & 51 & 10 \\
Intra & & & & \\
ADA- & 239.5 & 387 & 798 & 12 \\
Inter & & & & \\
\hline
\end{tabular}

\section{Appendix B}

The relevant deductions for obtaining Equation 10 are given below. The relevant deductions for Equations 14, 24 , and 28 can be obtained in an analogous way.

Based on the deduction in Appendix A, the total transmission time expectation of the traffic with $\gamma$ interactions for HMIPv6 in BT mode is also the sum of infinite geometric series:

$$
\begin{aligned}
E_{\mathrm{HMIPv6}}^{\mathrm{BT}}(T) & =\gamma \cdot E_{\mathrm{HMIPv6}}^{\mathrm{BT}}(D) \\
& +\gamma \cdot E_{\mathrm{HMIPv}}^{\mathrm{BT}}(D) \frac{E_{\mathrm{HMIPv}}^{\mathrm{BT}}(L)}{\alpha} \\
& +\gamma \cdot E_{\mathrm{HMIPv}}^{\mathrm{BT}}(D)\left(\frac{E_{\mathrm{HMIPv6}}^{\mathrm{BT}}(L)}{\alpha}\right)^{2}+\ldots
\end{aligned}
$$

The key problem is how to obtain the common ratio in this infinite geometric series.

Based on the definition of $\beta$, the expectation of handoff latency for HMIPv6 in BT mode can be given by:

$$
E_{\mathrm{HMIPv}}^{\mathrm{BT}}(L)=\frac{E_{\mathrm{HMIPv6}}^{\mathrm{BT}-\operatorname{INTER}}(L)+\beta \cdot E_{\mathrm{HMIPv} 6}^{\mathrm{BT}-\operatorname{INTA}}(L)}{\beta+1}
$$

Based on (B.2), (B.1) can be expressed as:

$$
\begin{aligned}
& E_{\mathrm{HMIPv6}}^{\mathrm{BT}}(T) \\
& =\frac{\gamma \cdot E_{\mathrm{HMIPv} 6}^{\mathrm{BT}}(D)}{1-\frac{E_{\mathrm{HMIPV} 6}^{\mathrm{BT}-\mathrm{INTER}}(L)+\beta \cdot E_{\mathrm{HMIPv} 6}^{\mathrm{BT}-\operatorname{INTRA}_{1}}(L)}{\alpha(\beta+1)}} \\
& =\frac{\alpha \cdot \gamma(\beta+1)(8 d+10 \Delta+4 \delta)}{\alpha \beta+\alpha-2 \beta \Delta-\beta \rho-4 d-8 \Delta-2 \delta-\rho}
\end{aligned}
$$

\section{List of abbreviations}

ADA: asymmetric double-agents; BT: bidirectional tunneling; BU: binding update; CMP: correspondent mobile proxy; CNs: correspondent nodes; CoA: care-of address; CoT: care-of test; CoTI: care-of test init; DHoA: distributed home address; FA: foreign agent; GFA: gateway foreign agent; HA: home agent; HoA_IR: home address for IP reachability; HoA_OR: home address for optimized routing; HoTI: home test init; HoT: home test; IRHA: reachability home agent; LCoA: on-link care-of address; LMP: local mobile proxy; MN: mobile node; MIPv4: mobile IPv4; MAP: mobility anchor point; NGWN: nextgeneration wireless networks; NAR: new access router; ORHA: optimized routing home agent; PAR: previous access router; RCoA: regional care-of address; RO: route optimization; RTTs: round-trip times; RR: return routability; TD: transmission delay; TA: temporary home agent.

\section{Acknowledgements}

This work has been supported by the National Basic Research Program of China (No. 2011CB302702), the National Natural Science Foundation of China (No. 60803140, No. 60970133, No. 61070187, and No. 61003225) and the Beijing Nova Program.

\section{Author details}

'Institute of Computing Technology, Chinese Academy of Sciences, Beijing, 100190, People's Republic of China ${ }^{2}$ Collaborative Computing Lab, Lenovo Corporate Research \& Development, Beijing, 100085, People's Republic of China ${ }^{3}$ Department of Electronic Engineering, Macquarie University, Sydney, 
NSW, 2109, Australia ${ }^{4}$ Graduate University of the Chinese Academy of Sciences, Beijing, 100049, People's Republic of China

\section{Competing interests}

The authors declare that they have no competing interests.

Received: 14 January 2011 Accepted: 30 June 2011

Published: 30 June 2011

\section{References}

1. C Makaya, S Pierre, An analytical framework for performance evaluation of IPv6-based mobility management protocols. IEEE Trans. Wireless Commun. 7(3), 972-983 (2008)

2. AH Zahran, B Liang, A Saleh, Signal threshold adaptation for vertical handoff in heterogeneous wireless networks. Mobile Netw. Appl. 11, 625-640 (2006)

3. E Fogelstroem, A Jonsson, C Perkins, Mobile IPv4 regional registration. Internet Engineering Task Force, RFC 4857, Jun. 2007

4. A Valko, Cellular IP: a new approach to internet host mobility. ACM SIGMOBILE Computer Comm. Rev. 29(1), 50-65 (1999)

5. A Misra, S Das, A Dutta, A McAuley, S Das, IDMP-based fast handoffs and paging in IP-based 4G mobile networks. IEEE Commun. Mag. 40(3), 138-145 (2002)

6. R Ramjee, K Varadhan, L Salgarelli, SR Thuel, SY Wand, T La-Porta, HAWAll: a domain-based approach for supporting mobility in wide-area wireless networks. IEEE/ACM Trans. Netw. 10(3), 396-410 (2002)

7. J Xie, IF Akyildiz, A distributed dynamic regional location management scheme for mobile IP. in Proceedings of IEEE INFOCOM 2002, pp. 1069-1078, June 2002

8. WC Ma, YG Fang, Dynamic hierarchical mobility management strategy for mobile IP networks. IEEE J. Selected Areas Commun. 22(4), 664-676 (2004)

9. CE Perkins, DB Johnson, Route optimization in mobile IP. Internet Draft (Work in Progress), Internet Engineering Task Force, draft-ietfmobileipoptim-11.txt., Sept. 2001

10. D Johnson, C Perkins, J Arkko, Mobility support in IPv6. Internet Engineering Task Force, RFC 3775, June 2004

11. C Perkins, IP mobility support for IPv4. Internet Engineering Task Force, RFC 3220, Jan. 2002

12. H Soliman, C Castelluccia, K EIMalki, L Bellier, Hierarchical mobile IPv6 (HMIPv6) mobility management. Internet Engineering Task Force, RFC 5380, Oct. 2008

13. K Weniger, MIPv6 correspondent node-targeted location privacy and optimized routing. Internet Draft (Work in Progress), Internet Engineering Task Force, draft-weniger-mobopts-mip6-cnlocpriv-03., Nov. 2008

14. If Akyildiz, J Xie, S Mohanty, A survey on mobility management in next generation all-IP based wireless systems. IEEE Wireless Commun. 11(4), 16-28 (2004)

15. N Kara, Mobility management approaches for mobile IP networks: performance comparison and use recommendations. IEEE Trans Mobile Comput. 8(10), 1312-1325 (2009)

16. J Cao, L Zhang, H Chan, SK Das, Design and performance evaluation of an improved mobile IP protocol. in Proceedings of IEEE INFOCOM 2004, pp. 319-329, Mar. 2004

17. R Koodli, Mobile IPv6 fast handovers. Internet Engineering Task Force, RFC 5268, June 2008

18. YJ Lee, IF Akyildiz, A new scheme for reducing link and signaling costs in mobile IP. IEEE Trans Comput. 52(6), 706-711 (2003)

19. R Zheng, Y Ge, JC Hou, SR Thuel, A case for mobility support with temporary home agents. ACM SIGMOBILE Mob Comput Commun Rev. 6(1), 32-46 (2002)

20. Y Mao, B Knutsson, HH Lu, JM Smith, DHARMA: distributed home agent for robust mobile access. in Proceedings of IEEE INFOCOM 2005, pp. 1196-1206, Mar. 2005

21. X P'erez-Costa, M Torrent-Moreno, H Hartenstein, A performance comparison of mobile IPv6, hierarchical mobile IPv6, fast handovers for mobile IPv6 and their combination. ACM SIGMOBILE Mob Comput Commun Rev. 7(4), 5-19 (2003)

22. Y Gwon, J Kempf, A Yegin, Scalability and robustness analysis of mobile IPv6, fast mobile IPv6, hierarchical mobile IPv6, and hybrid IPv6 mobility protocols using a large-scale simulation. in Proceedings of IEEE ICC 2004, pp. 7. 4087-4091, June 2004
23. X P'erez-Costa, R Schmitz, H Hartenstein, M Leibsch, A MIPv6, FMIPv6 and HMIPv6 handover latency study: analytical approach. IST Mobile Wireless Commun Summit. 100-105 (2002)

24. C Castelluccia, HMIPv6: a hierarchical mobile IPv6 proposal. ACM SIGMOBILE Mob. Comput Commun. Rev. 4(1), 48-59 (2000)

25. S Pack, Y Choi, Performance analysis of fast handover in mobile IPv6 networks. IFIP Pers Wireless Commun. LNCS 2775, 679-691 (2003)

26. S Pack, Y Choi, A study on performance of hierarchical mobile IPv6 in IPbased cellular networks. IEICE Trans Commun. E87-B(3), 462-469 (2004)

27. HY Jung, EA Kim, JW Yi, HH Lee, A scheme for supporting fast handover in hierarchical mobile IPv6 networks. ETRI J. 27(6), 798-801 (2005)

28. G Giaretta, J Kempf, V Devarapalli, Mobile IPv6 bootstrapping in split scenario. Internet Engineering Task Force, RFC 5026, Oct. 2007

29. MobiWan: NS-2 extensions to study mobility in Wide-Area IPv6 Networks. http://www.inrialpes.fr/planete/mobiwan/

30. The Network Simulator - ns-2, http://www.isi.edu/nsnam/ns/

\section{doi:10.1186/1687-1499-2011-25}

Cite this article as: Liu et al: Low latency IP mobility management: protocol and analysis. EURASIP Journal on Wireless Communications and Networking 2011 2011:25.

\section{Submit your manuscript to a SpringerOpen ${ }^{\circ}$ journal and benefit from:}

- Convenient online submission

- Rigorous peer review

- Immediate publication on acceptance

- Open access: articles freely available online

- High visibility within the field

- Retaining the copyright to your article

Submit your next manuscript at $\gg$ springeropen.com 